\title{
Sound Source Extension Library for Modelica
}

\author{
Johann Emhofer Raimund Zitzenbacher Christoph Reichl \\ Center for Energy, AIT Austrian Institute of Technology, Giefinggass 2, 1210 Wien, Austria. \\ \{johann.emhofer, raimund.zitzenbacher.fl, christoph.reichl\}@ait.ac.at
}

\begin{abstract}
Transient thermodynamic models in Modelica are widely used for energetic simulations of machines and systems which are located nearby people. Nevertheless, so far no libraries exist which consider the noise of such machines in the simulations. The Sound Source Extension library (SSElib) proposed in this work, should close this gap. With the aid of the SSElib, acoustic characteristics can be added to existing Modelica models (e.g. to a compressor or a pump model). The acoustic characteristic added to the existing model is frequency dependent in the one-octave band and could further depend on an input parameter like the rotational speed of a compressor. With the inclusion of sound sources into energetic models, the sound behavior of machines can be considered and control strategies can be optimized to lower the noise of machines.
\end{abstract}

Keywords: Modelica, sound, noise, acoustics, heat pump

\section{Introduction}

Due to local mechanical vibrations of a component in air, local displacements of the air atoms and changes in local pressure or density are excited. The local changes propagate to the neighboring atoms. The propagation of these vibrations are better known as sound which can be recognized by the human ear. Speech, music or acoustic signals are wanted effects of sound, whereas the noise of machines are experienced as disturbing. The aim of the Sound Source Extension Library (SSElib) is to consider the unwanted noise excited from machines. With the SSElib one can extend existing Modelica components with a sound source that can depend on a variable of the component itself. This variable could be a frequency of a fan, the pressure drop over a heat exchanger or any other variable that influences the sound characteristic of the machine. In other words, an acoustic characteristic which depends on the operating conditions of a component can be added to a new or existing component.

In the SSElib we use basic acoustic calculation methods to estimate the all over loudness of a machine. Note that the main aim of the library is not an accurate prediction of the loudness but it should show how the operation point of a machine influences their noise emission. Hence, solely simple correlations for noise propagation and reduction are included in the library to give a first hint about how the acoustic characteristic of machines behave. The strength of the SSElib is the easy integration into existing models without the need of significant extra computational power in Modelica.

Several mature methods like Finite Element Methods (FEM), Boundary Element Methods (BEM) or Computational Aero Acoustics (CAA) exist for accurate sound propagation calculations and therefore such methods should be used if a detailed sound analysis is needed. A good overview of these methods can be found in (Crocker, 2007).

The SSElib was developed in Dymola 2016 and testing was performed within the Testers and Examples package of the library. The SSElib builds on the Modelica Standard Library (MSL) and no additional libraries are needed. Most of the Testers were also tested in OpenModelica 1.9.6 without any problems. Besides this publication, a UsersGuide package was added to the library on top level, to help users with the implementation of the SSElib into their models.

SSElib is published under the Modelica License 2.

\section{Methodology}

\subsection{General}

The following assumptions were made in order to keep the equations simple:

- All sound sources are independent point sources.

- The sound fields considered in the SSElib are assumed to be diffuse and incoherent in all frequencies.

- The noise source volume has to be less than about 0.3 to 0.4 of an enclosure volume for calculations of damping (Crocker, 2007). If the noise source occupies more than a third of the enclosed volume of the sealed enclosure, the sound field is neither reverberant nor diffuse. Nevertheless, the discussed methods will be used as a first approximation of insertion losses in an enclosure even if this requirement is not fulfilled.

In this work, we concentrate on the sound pressure $p$ and the sound power $W$. The sound pressure is always connected to a location and describes the local pressure amplitude at this location. Therefore, the sound pressure depends on the distance from the sound source $\left(\propto 1 / r^{2}\right.$ for a point source) and the symmetry of the emitted sound waves from the source (monopole, dipole, etc.). From 
the sound pressure one can calculate the sound intensity $I$ which takes the material properties of the sound propagating media into account. For both, a radial symmetric point source and a plane wave moving in one direction, this gives (Crocker, 2007):

$$
I=\frac{p_{r m s}^{2}}{\rho c}
$$

for the time averaged sound intensity, where $\rho$ is the density of the medium (air) and $c$ is the speed of sound in the medium. In the SSElib, the denisty of air and the speed of sound in air are assumed as constant values with $1.204 \mathrm{~kg} / \mathrm{m}^{3}$ and $343 \mathrm{~m} / \mathrm{s}$, respectively. Subsequently, the sound power $W$ of a sound source can be calculated from integrating the sound intensity over an enclosing surface around the sound source in a far field assumption:

$$
W=\int_{S} \frac{p_{\mathrm{rms}}^{2}}{\rho c} \mathrm{~d} S=\frac{1}{\rho c} \sum_{j} p_{\mathrm{rms}, j}^{2} S_{j}
$$

where $p_{\text {rms }, j}$ is the sound pressure and $S_{j}$ is a partial area of the enclosing surface where constant sound pressure $p_{\text {rms }, j}$ is assumed. From (2) one can see, that if two variables from $(W, p, S)$ are known, the third can be calculated. For unsymetrical sound sources, the sound pressure on the enclosing surface becomes rapidly complex, hence only two special cases are considered in the SSElib: a spherical propagation and a one dimensional propagation. For both one finds:

$$
W=\frac{1}{\rho c} p_{\mathrm{rms}}^{2} S
$$

where $S$ represents the surface through which the sound propagates and $p_{\text {rms }}$ is the effective sound pressure at $S$.

If a point source is located in the free room, $S$ would be $4 \pi r^{2}$ where $r$ is the radial distance from the point source. If a rigid surface is located below the point source, the sound will only propagate to a spherical half space with a surface of $2 \pi r^{2}$, therefore $S$ is only half of the free room situation. For a sound source standing on a rigid surface in front of a wall and for a sound source located in a corner, $S$ would even be a fourth or an eight compared to the free room situation. In other words, for a given sound power, the sound pressure could be significantly higher for different installation situations compared to the free room situation, due to the fact that the kinetic energy generated by the sound source has to be transported through a smaller surface (c.f. Table 1).

Contrary to the sound pressure, the sound power is a characteristic value of the sound source which is independent of the location or the symmetry of the sound source. Hence, once the sound power level is known, the sound pressure level can be calculated for known geometries.

Usually both the sound pressure level and the sound power level of a single frequency source, are given in log- arithmic $\mathrm{dB}$ units:

$$
\begin{array}{ll}
L_{p}(\mathrm{~dB})= & 20 \log \left(\frac{p}{p_{0}}\right) \\
L_{W}(\mathrm{~dB})= & 10 \log \left(\frac{W}{W_{0}}\right)
\end{array}
$$

where $p_{0}$ is the absolute threshold of hearing at $2 \times 10^{-5} \mathrm{~Pa}$ and $W_{0}$ is $10^{-12} \mathrm{~W}$.

Combining (3),(4),(5) and concerning that $W_{0}=$ $p_{0}^{2} /(\rho c)$, leads to a direct link between $L_{p}$ and $L_{W}$ :

$$
L_{p}=L_{W}-10 \log \left(\frac{S}{1 \mathrm{~m}^{2}}\right)
$$

As already noticed in (3), a change of the enclosed surface through which the sound from the sound source propagates leads to a reduction or an enhancement of the sound pressure level. From (6) follows for two different locations $A$ and $B$ :

$$
L_{p, B}=L_{p, A}-10 \log \left(\frac{S_{B}}{S_{A}}\right)
$$

where $S_{A}$ and $S_{B}$ are two different enclosing surfaces with constant sound pressure level $L_{p, A}$ and $L_{p, B}$, respectively. If the enclosed surface increases e.g. if the distance between sound source and observer increases, the sound pressure level decreases. If the enclosed surfaces decreases e.g. at the inlet into a duct, the sound pressure level increases.

For a radial symmetric point source (6) leads to an equation where $S$ can be described with the radial distance $r$ directly:

$$
L_{p}=L_{W}-20 \log \left(\frac{r}{1 \mathrm{~m}}\right)-10 \log \left(\frac{4 \pi}{\rho c} \frac{p_{0}^{2}}{W_{0}}\right)
$$

The last term is dominated by $10 \log (4 \pi)$ and is usually approximated with $11 \mathrm{~dB}$ in various textbooks.

The difference of two sound pressure levels of a constant radial symetrical point source changes with the distance. From (8) follows for two different locations $A$ and $B$ :

$$
L_{p, B}=L_{p, A}-20 \log \left(\frac{r_{B}}{r_{A}}\right)
$$

where $r_{A}$ and $r_{B}$ are the different distances to the point source. Hence, doubling the distance leads to a reduction of $6 \mathrm{~dB}$. Note that doubling the distance in (9) is equivalent to quadruple the surface of constant sound pressure.

As already discussed, $p_{\text {rms }}^{2}$ will be doubled if the sound source stands on a rigid floor and the same sound power level of the source is assumed. Hence, the sound pressure level will be enhanced by $3 \mathrm{~dB}$. Similar considerations lead to an enhancement of $6 \mathrm{~dB}$ and $9 \mathrm{~dB}$ for a sound source standing on a rigid surface in front of a wall and a sound source in a corner, respectively. Therefore, simple rules as summarized in Table 1 hold for these scenarios. 
Table 1. Noise enhancement for different installation situations

\begin{tabular}{lccc}
\hline Situation & $\Delta L(\mathrm{~dB})$ & $\Delta S$ \\
\hline floor & & +3 & $S_{\text {free }} / 2$ \\
wall and floor & & +6 & $S_{\text {free }} / 4$ \\
corner & & +9 & $S_{\text {free }} / 8$ \\
\hline
\end{tabular}

\subsection{Frequency analysis}

Up to this point, only single frequency sources were considered. But both, sound pressure and power can be broken down into frequency bands as shown by Fourier over 200 years ago. It is common in acoustics to divide the frequency spectrum into frequency bands like the one-octave ot the one-third-octave band. For an octave band the lower and upper cutoff frequencies $\left(f_{1}\right.$ and $\left.f_{\mathrm{u}}\right)$ are defined as:

$$
f_{1}=f_{\mathrm{c}} / \sqrt{2} ; f_{\mathrm{u}}=\sqrt{2} f_{\mathrm{c}}
$$

where $f_{\mathrm{c}}$ is the center frequency of the band. From (10) follows the center frequency:

$$
f_{\mathrm{c}}=\sqrt{f_{1} f_{\mathrm{u}}}
$$

Furthermore, from (10) follows that the upper cut off frequency is always twice the lower cut off frequency $f_{\mathrm{u}}=$ $2 f_{1}$ and that the bandwidth is $\Delta f=\sqrt{2} f_{\mathrm{c}}$. For the i-th frequency band, the center frequency follows:

$$
f_{\mathrm{c}, i}=2^{(i-1)} f_{\mathrm{c}, 1}
$$

where $f_{\mathrm{c}, 1}$ is the first center frequency $(15.625 \mathrm{~Hz}$ in the one-octave band).

Considering frequency analysis, the frequency dependent sound pressure level $\mathbf{L}_{\mathbf{p}}$ and the sound power $\mathbf{L}_{\mathbf{W}}$ are generally described as vectors in the SSElib:

$$
\mathbf{L}_{\mathbf{p}}=\left(\begin{array}{c}
L_{\mathrm{p}, 1} \\
\vdots \\
L_{\mathrm{p}, i} \\
\vdots \\
L_{\mathrm{p}, n}
\end{array}\right), \quad \mathbf{L}_{\mathbf{W}}=\left(\begin{array}{c}
L_{\mathrm{W}, 1} \\
\vdots \\
L_{\mathrm{W}, i} \\
\vdots \\
L_{\mathrm{W}, n}
\end{array}\right)
$$

where each row corresponds to a frequency band with a center frequency $\mathbf{f}_{\mathbf{c}}=\left(f_{\mathrm{c}, 1} \ldots f_{\mathrm{c}, \mathrm{i}} \ldots f_{\mathrm{c}, \mathrm{n}}\right)^{\mathrm{T}}$. The values of $\mathbf{f}_{\mathbf{c}}$, as well as the number of rows $n$ depend on the chosen frequency band. E.g. if the octave band is choosen, the center frequencies are $\mathbf{f}_{\mathbf{c}}=(16,31.5,63,125,250,500,1 \mathrm{e} 3,2 \mathrm{e} 3,4 \mathrm{e} 3,8 \mathrm{e} 3,16 \mathrm{e} 3)^{\mathrm{T}} \mathrm{Hz}$ and therefore $n=11$ (c.f. Table 4). The logarithmic sum of the sound pressure levels at different center frequencies gives then the total sound pressure level and the total sound power level, respectively:

$$
\begin{aligned}
& L_{\mathrm{p}, \text { total }}(\mathrm{dB})=10 \log \left(\sum_{i=1}^{n} 10^{L_{\mathrm{p}, \mathrm{i}} / 10}\right) \\
& L_{\mathrm{W}, \text { total }}(\mathrm{dB})=10 \log \left(\sum_{i=1}^{n} 10^{L_{\mathrm{W}, \mathrm{i}} / 10}\right)
\end{aligned}
$$

The same addition rules (13) and (14) are valid if the sound pressure or sound power levels of independent sound sources have to be added to a total sound pressure or sound power level, respectively.

The relative loudness of sound that can be perceived by the human ear is usually calculated by weighting the instrument-measured sound levels with a frequency dependent curve or table. The most common used curve is the A-weighting curve which is defined in several national and international standards, like the (IEC 61672-1, 2003). This curve dampens the sound at low and high frequencies whereas the intermediate frequencies stay unfiltered or are slightly enhanced. Table 4 in the Appendix shows the weighting coefficients $\Delta_{\mathrm{i}}$ used for A-weighting at different center frequencies in the one-octave band which are used in the SSElib. If the one-octave band is chosen, the weighting coefficient vector is $\Delta_{\mathrm{A}}=(-56.7,-39.4,-$ $26.2,-16.1,-8.6,-3.2,0,1.2,1.0,-1.1,-6.6)^{\mathrm{T}} \mathrm{dB}$.

The A-weighted total sound pressure level can then be calculated with:

$$
\begin{gathered}
\tilde{L}_{\mathrm{p}, \text { total }}(\mathrm{dBA})=10 \log \left(\sum_{i=1}^{n} 10^{\left(L_{\mathrm{p}, \mathrm{i}}+\Delta_{\mathrm{A}, i}\right) / 10}\right) \\
\tilde{L}_{\mathrm{W}, \text { total }}(\mathrm{dBA})=10 \log \left(\sum_{i=1}^{n} 10^{\left(L_{\mathrm{W}, \mathrm{i}}+\Delta_{\mathrm{A}, i}\right) / 10}\right)
\end{gathered}
$$

It is common, that A-weighted sound levels are described with the unit $\mathrm{dBA}$ or $\mathrm{dB}(\mathrm{A})$.

One has to be aware if data is given in the unit $\mathrm{dB}$ or $\mathrm{dB}(\mathrm{A})$. However, with (15) one can always convert the units to each other.

\subsection{Noise reduction}

Noise reduction can be achieved with several active and passive methods. An active method could be to operate a machine in a silent operating point. Such points can be found by extending simulation models with the SSElib. A passive method is the integration of sound absorbing materials or silencers. Currently, two main noise reduction methods are implemented in the SSElib. The first one describes the reduction with frequency dependent differences on the sound pressure or power level. Such a description is often used for silencers in ducts. Manufacturers usually provide these data to their customers. Table 5 in the Appendix shows typical values for the differences in sound pressure level $\Delta L_{\mathrm{s}}$ in a $60 \times 60 \mathrm{~cm}$ rectangular silencer with a length of $50 \mathrm{~cm}$. Using the vector notation one can easily describe the noise reduction with:

$$
\mathbf{L}_{\mathbf{p}, \text { out }}=\mathbf{L}_{\mathbf{p}, \text { in }}+\Delta \mathbf{L}_{\mathrm{s}}
$$


where $\mathbf{L}_{\mathbf{p}, \text { out }}$ is the frequency dependent sound pressure level at the outlet of the silencer, $\mathbf{L}_{\text {in }}$ is the frequency dependent sound pressure level at the inlet of the silencer and $\Delta \mathbf{L}_{\mathrm{s}}$ has to be taken from manufacturers data or text books. Note that according to (6) the reduction of $\mathbf{L}_{\mathbf{p}}$ in (17) goes hand in hand with a reduction of the sound power level $\mathbf{L}_{\mathrm{W}}$ as sound power is converted to heat in the absorber material.

The other noise reduction method implemented in the SSElib uses absorbing material mounted in the enclosure of a machine or on the enclosing walls of a room. As the assumed sound fields are diffuse and reverberant the noise in the enclosure can be described with an average sound pressure level inside the enclosure. For this, we have to introduce frequency dependent sound absorption coefficients $\alpha$ for given surfaces and center frequencies. The absorbed sound power is therefore given with:

$$
W_{a b s}=\frac{\tilde{p}^{2}}{4 \rho c} \sum_{j} \alpha_{j} S_{j}
$$

where $\tilde{p}$ is the diffuse sound pressure in the enclosure, $\tilde{p}^{2} /(4 \rho c)$ represents the equivalent power per $\mathrm{m}^{2}$ and $S_{j}$ and $\alpha_{j}$ are partial surfaces and their corresponding absorption coefficients, respectively. The term $\sum_{j} \alpha_{j} S_{j}$ is often referred to as the equivalent area of an open window in an enclosure with no absorption.

In the steady-state, the absorbed sound power has to be equal to the sound power propagating from the sound source inside the enclosure. Hence, $\mathbf{W}_{\mathbf{a b s}}$ is equal to $\mathbf{W}$. From (18) it is obvious that the sound pressure $\tilde{p}$ becomes smaller for higher absorption coefficients or higher surface areas. Using logarithmic notations one finds (see also (Möser, 2012)):

$$
\mathbf{L}_{\tilde{\mathbf{p}}}=\mathbf{L}_{\mathbf{W}}-10 \log \left(\frac{\sum_{j} \boldsymbol{\alpha}_{j} S_{j}}{1 \mathrm{~m}^{2}}\right)+6 \mathrm{~dB}
$$

Dependent on the material and the geometry of the enclosure, a part of the absorbed sound power is converted to thermal energy whereas the other part remains sound and will be radiated from the outer surface of the enclosure. Similar to $\boldsymbol{\alpha}$, the transmission can be described by frequency dependent transmission coefficients $\tau$ :

$$
\mathbf{W}_{\tau}=\mathbf{W} \circ \frac{\sum_{j} \tau_{j} S_{j}}{\sum_{j} S_{j}}
$$

where $\mathbf{W}_{\tau}$ is the sound power propagating from the outer surface of the enclosure.

\section{Implementation}

\subsection{Connectors}

The acoustic port $(\bullet)$ in the SSElib consists of two variables namely, the frequency dependent sound power $\mathbf{W}$ (flow variable) and the enclosing surface $S$ at the ports location.
Although logarithmic units are common in sound calculations, decimal units were used to describe the sound power to circumvent problems if the flow direction is unknown. Since also negative logarithmic sound levels have still a positive sound power, the descriptions with flow variables was not possible without introducing a further variable. Therefore, and for the sake of simplicity decimal units are used in the SSElib to describe the sound power in the connectors. Currently, only the one-octave band is implemented in the SSElib, hence the sound power $\mathbf{W}$ and the center frequencies $\mathbf{f}_{\mathbf{c}}$ are described as vectors with 11 rows according to the one-octave band (c.f.Table 4). The center frequency vector $\mathbf{f}_{\mathbf{c}}$ is located in the Constants package.

\subsection{Components}

Several components were realized in the SSElib. Table 2 gives an overview of the components and links them to the

\begin{tabular}{|c|c|c|}
\hline Name & & Reference \\
\hline AcousticSource & & - \\
\hline AcousticSink & $\odot$ & - \\
\hline Add & 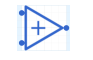 & $(13),(14)$ \\
\hline Converter_dBA & sisosent & $(15),(16)$ \\
\hline InstallationSituationFloor & $\bullet$ & Table 1 \\
\hline InstallationSituationWall & $\cdot$ & Table 1 \\
\hline InstallationSituationCorner & $\bullet$ & Table 1 \\
\hline RadialDistance & 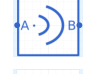 & $(8),(9)$ \\
\hline OpenWindow & $((\cdot)))$. & (6) \\
\hline Silencer & 르 & (17) \\
\hline Enclosure & ((o) & $(18),(19)$ \\
\hline EnclosureWithTransmission & $(\infty)$ & (18),(19),(20) \\
\hline
\end{tabular}
equations and tables used in this work.

Table 2. Components of the SSElib

\subsection{Sensors}

In order to observe the sound pressure and sound power levels at different locations inside the models, four sensors are provided in the library which can be connected in between an acoustic connection. For both, the sound power and the sound pressure sensors, an unfiltered version and an A-weighted version exist. Table 3 shows the four sensors. Please note that we have used $\mathrm{dB}$ instead of $\mathrm{dB}(\mathrm{A})$ or $\mathrm{dBA}$ as unit for the A-weighted variables. The reason is that $\mathrm{dB}(\mathrm{A})$ or $\mathrm{dBA}$ aren't valid units in Modelica. 
Table 3. Sensors of the SSElib

\begin{tabular}{llc}
\hline Name & Reference \\
\hline SoundPressureSensor & $(4),(6),(13)$ \\
SoundPressureSensor(dBA) & $(4),(6),(15)$ \\
SoundPowerSensor & & $(5),(6),(14)$ \\
SoundPowerSensor(dBA) & & $(5),(6),(16)$ \\
\hline
\end{tabular}

\subsection{Sound Source Extension}

The main task of the SSElib is to provide a model to extend existing models with acoustic characteristics. This model is the AcousticExtensionOneOctave model (10cated in the SoundSourceExtension package. It should be used in the following way:

- Create a model which represents your new acoustic component

- Extend your new model with the sound source extension from the SSElib

- Extend your new model with your non-acoustic component e.g. a fluid pump

- Use your new model in the simulations instead of the old model and modify the acoustic parameters to match your components sound characteristic. Don't forget to connect your new model to at least one acoustic sink.

A new model for an extended fluid pump was implemented in the following way using the PrescribedPump model from the MSL (Modelica.Fluid.Machines.PrescribedPump):

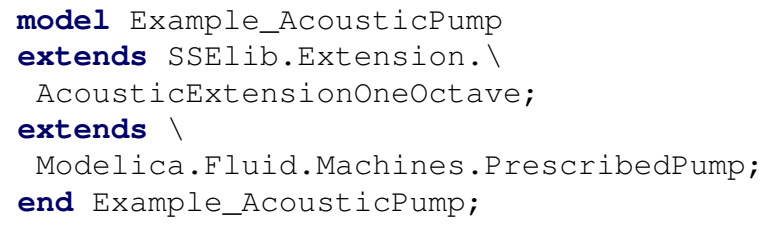

The extended pump can be found in the TestersAndExamples package. Figure 1 shows the graphical representation of the pump from the MSL extended with a sound source.

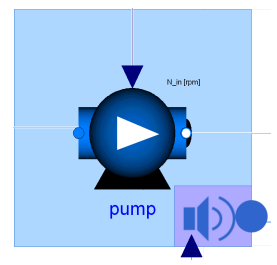

Figure 1. Fluid pump from the MSL extended with a sound source.
Contrary to the connections of the original pump model, the new model has now an additional real input and a sound source connector as depicted in the lower right corner. The additional input is simply called "soundInput" and can be used to influence the sound source levels. In the case of the pump, the rotational speed of the pump would be a reasonable input variable, due to the fact that the acoustic characteristic of the pump is expected to change significantly with the pump speed.

Figure 2 shows artificial sound data for a pump. We assume that sound power level at the rotational speeds $n=$ $30,50,70$ and $90 \mathrm{~Hz}$ have been measured and should be used for the simulations. They are depicted as bar plots in Fig. 2. In order to estimate the correct sound pressure levels at different rotational speeds, one could use the measured data directly in Modelica as table data and calculate the corresponding sound power levels with the aid of interpolation functions. As this method would lead to significant loss of time, we propose to use polynomial functions to describe the sound power data. The surface plot in Fig. 2 shows a polynomial function which was fitted to the data using polynomial functions from the Numerical Python library NumPy (Oliphant, 2006).

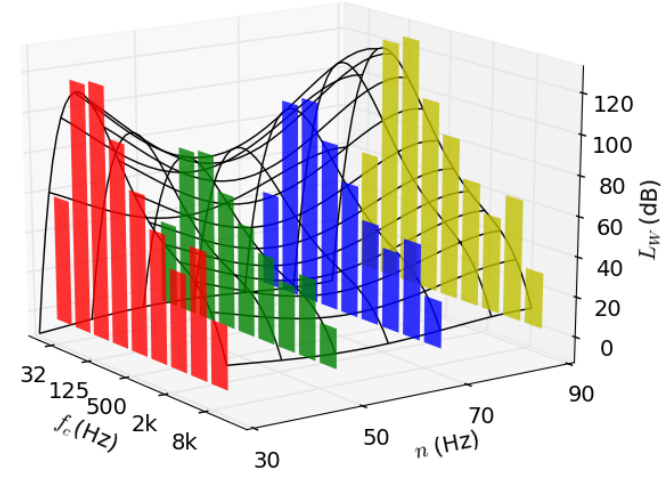

Figure 2. Sound power $L_{\mathrm{W}}$ dependency on the rotational speed of the pump $n$ and the frequency bands around the center frequency $f_{c}$. The surface around the bar plots represents a polynominal fit.

To use polynomial functions, polynomial coefficients have to be passed to the extended model as a parameter. In general these coefficients $c$ should have the form:

$$
L_{\mathrm{W}}(i, n)=\sum_{k, l} c_{k, l} i^{k} n^{l}
$$

where $i$ is the frequency band of the octave band (c.f. Table 4) and $n$ is the rotational speed of the pump. Note that we used the integer of the frequency band instead the center frequency on purpose, as it is easier to fit the polynomial functions into data with linear distributed nodes. Currently, the SSElib only supports polynomials with $k=l=3$ or $k=l=4$. 


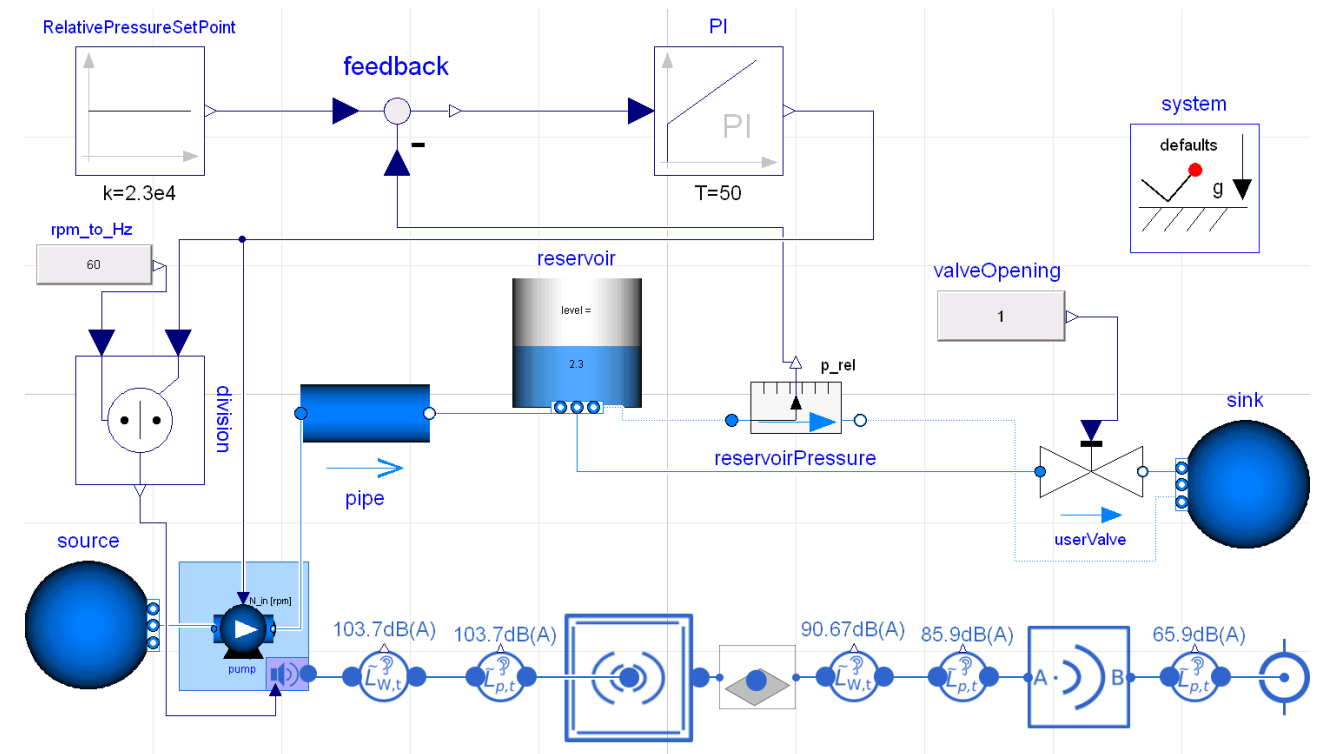

Figure 3. System sketch of the SSElib example SSElib.TestersAndExamples.Example.

\subsection{Testers and Examples}

Each component of the library has a testing model located in the Testers and Examples package. Furthermore, there is one extended component (Example_AcousticPump, Fig. 1) and one example (Example, Fig. 3) located inside this package.

The example was derived from the PumpingSystem example of the MSL, originally written by Francesco Casella.

Contrary to the original example the current pump can operate at different pump speeds and is controlled by a not very well designed PI-controller. Furthermore, the pump was extended with an acoustic sound source (c.f. section 3.4). A water pump which is sound-optimized for an operation around $50 \mathrm{~Hz}$ or $3000 \mathrm{rpm}$ was assumed and polynomial coefficients were used to represent this behavior (c.f. Fig. 2).

The A-weighted sound pressure level close to the machine ( $1 \mathrm{~m}$ distance) can be observed with SensorMachine.L_p_total_dBA and the speed of the pump can be observed with pumps. $N \_$in and pumps.soundInput in rpm and $\mathrm{Hz}$, respectively (Fig. 4). Starting from the soundoptimized speed of $3000 \mathrm{rpm}$ or $50 \mathrm{~Hz}$, the pump speed decreases to around $2260 \mathrm{rpm}$ or $38 \mathrm{~Hz}$ in the steady state after around $1200 \mathrm{~s}$. Simultaneously, the sound pressure level increases from $88 \mathrm{~dB}(\mathrm{~A})$ to $103 \mathrm{~dB}(\mathrm{~A})$ (red line in Fig. 4).

From an engineers point of view several options exists to lower the noise of the pump. One option could be, that the sound pressure measured at the machine or simulated on a computer is used to optimize the control strategy of the system. Another option could be to install sound absorption measures. The effect of the latter measure can be estimated by adding components from the SSElib.

In this example the second option is real- ized. The pump was covered with an enclosure (EnclosureWithTransmission) which stands on a floor without walls nearby (InstallationSituationFloor). An observer located $10 \mathrm{~m}$ away from the pump (RadialDistance) finally hears the pump with $66 \mathrm{~dB}(\mathrm{~A})$ in the steady state (c.f. SensorObserver.L_p_total_dBA in Fig. 4).
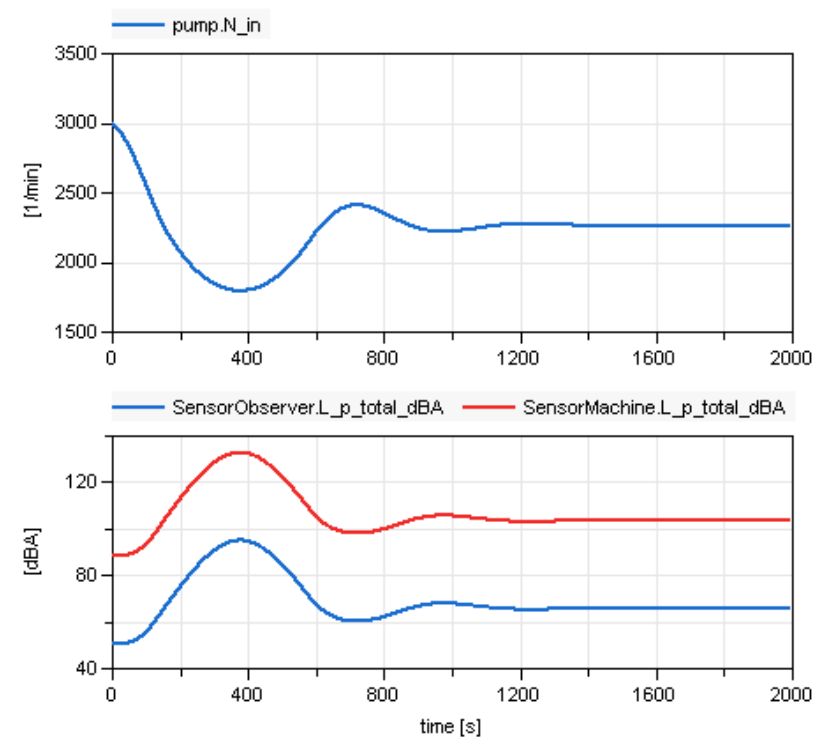

Figure 4. Time dependent pump speed (top) and time dependent total sound pressure level $L_{p, t o t a l}$ in dBA at different locations.

\subsection{Heat pump example}

Figure 5 shows a further example, where an air source heat pump, realized with the aid of the TIL library (TLKThermo $\mathrm{GmbH}$ ) and self-written components, was extended with sound sources. Please note, that this example is not included in the SSElib. 


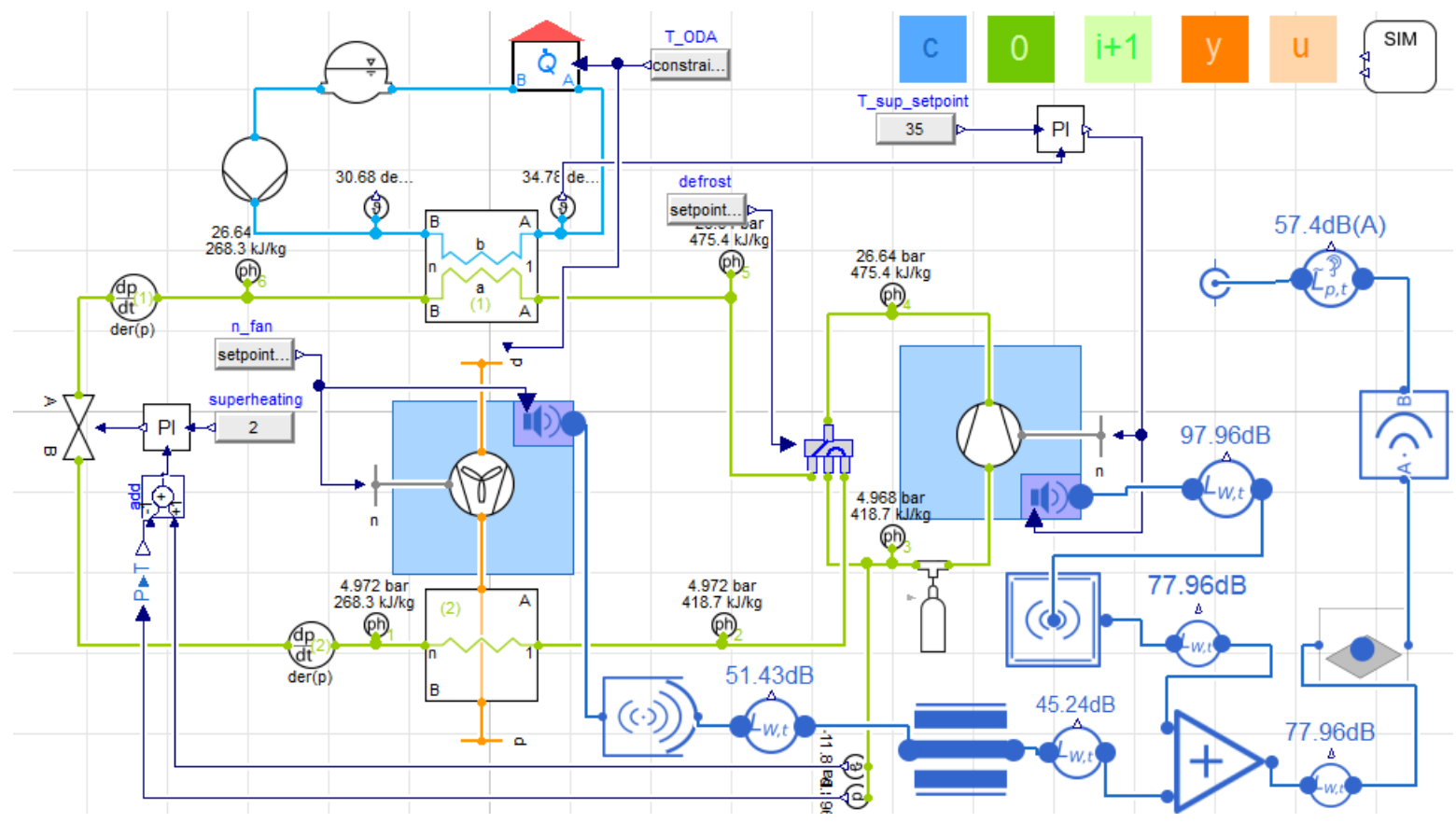

Figure 5. Air source heat pump model with an additional characteristic for the compressor and the fan. The components of the SSElib are mainly located at the lower right corner of the figure.

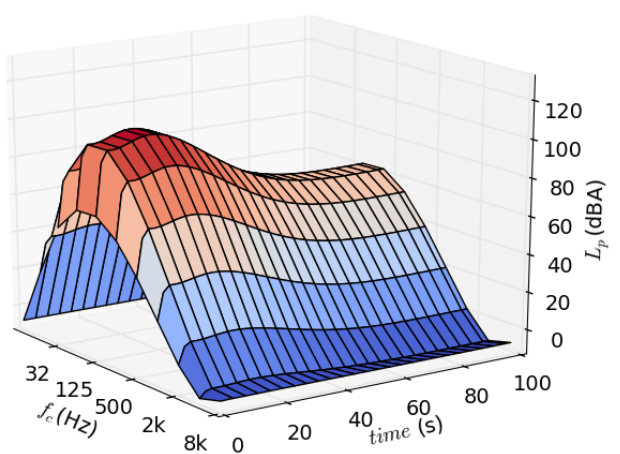

Figure 6. Transient behavior of the heat pump in the one-octave band.

The fan is housed in a casing with a $0.6 \mathrm{~m} \times 0.6 \mathrm{~m}$ opening, which is represented by the OpenWindow component. At the outlet of the casing a silencer (Silencer) is connected. The compressor is inside an casing without any free opening (EnclosureWithTransmission). The transmission coefficient to the outside for all frequencies is assumed with $\tau=0.01$. Both sound sources are located on a floor (InstallationFloor) and an observer (SoundPressureSensor_dBA) is $10 \mathrm{~m}$ away (RadialDistance). The total sound pressure level that the observer hears is about $57.4 \mathrm{~dB}(\mathrm{~A})$ at the operating point.

Figure 6 shows the transient behavior of the sound pressure level at the observer location. After starting the heat pump at time $=0$, the controller needs about $100 \mathrm{~s}$ to reach a steady-state condition. During this time, the sound power level reaches a significant maximum around $20 \mathrm{~s}$. In a sound optimized heat pump such peaks in the sound pressure level could be easily avoided if the controller considers this peaks at the start of the machine.

\section{Outlook}

The presented work shows the first version of a library which should continuously grow in the years to come. The following features should be implemented soon:

- Frequency resolutions in the one-third octave band

- Additional description methods for the acoustic behaviour besides the description with polynomials and constants.

- Validation of the models with measurement on an air source heat pump in AITs acoustic lab.

\section{Acknowledgment}

The Austrian Research Promotion Agency (FFG) is gratefully acknowledged for funding this work within the SilentAirHP project under Grant No. 848891. Furhtermore, we thank TLK-Thermo for technical advice.

\section{References}

M. J. Crocker, editor. Handbook of Noise and Vibration Control. Wiley, New Jersey, 2007.

IEC 61672-1. Electroacoustics - Sound level meters Part 1: Specifications ( International Electrotechnical Commission Standard No. 61672-1). Onlline on: 
https://webstore.iec.ch/publication/5708, last visited: 201703-07, 2003.

M. Möser. Technische Akustik. Springer-Verlag, Berlin Heidelberg, 9th edition, 2012. doi:10.1007/978-3-642-30933-5.

Travis E. Oliphant. Guide to NumPy. Provo, UT, March 2006. URL http: / / www. tramy.us/.

TLK-Thermo GmbH. TIL suite and TIL media: Commercial library for steady-state and transient simulation of thermodynamic systems such as heat pump,refrigeration, a/c, cooling and Rankine systems. Onlline on: https://www.tlkthermo.com/, last visited: 2017-01-22.

\section{Appendix}

Table 4. Frequency band index $i$, center frequencies $f_{\mathrm{c}}$ and Aweighting coefficients $\Delta_{\mathrm{A}, \mathrm{i}}$ for the one-octave band. The coefficients were taken from (IEC 61672-1, 2003)

\begin{tabular}{ccc}
\hline$i$ & $f_{\mathrm{c}}(\mathrm{Hz})$ & $\Delta_{\mathrm{A}, \mathrm{i}}(\mathrm{dB})$ \\
\hline 1 & 16 & -56.7 \\
2 & 31.5 & -39.4 \\
3 & 63 & -26.2 \\
4 & 125 & -16.1 \\
5 & 250 & -8.6 \\
6 & 500 & -3.2 \\
7 & 1000 & 0 \\
8 & 2000 & 1.2 \\
9 & 4000 & 1 \\
10 & 8000 & -1.1 \\
11 & 16000 & -6.6 \\
\hline
\end{tabular}

Table 6. Typical values for the sound absorption coefficient $\alpha$ of $5 \mathrm{~cm}$ thick melamine foam taken from the data sheet of a commercial available noise insulation material

\begin{tabular}{cc}
\hline center frequency $f_{\mathrm{c}}(\mathrm{Hz})$ & $\alpha(-)$ \\
\hline 125 & 0.13 \\
250 & 0.41 \\
500 & 0.6 \\
1000 & 0.9 \\
2000 & 0.85 \\
4000 & 0.93 \\
\hline
\end{tabular}

Table 5. Typical values for the differences in sound pressure level $\Delta L_{\mathrm{s}}$ in a $60 \times 60 \mathrm{~cm}$ rectangular silencer with a length of $50 \mathrm{~cm}$ taken from the data sheet of a commercial available silencer.

\begin{tabular}{cc}
\hline center frequency $f_{\mathrm{c}}(\mathrm{Hz})$ & $\Delta L_{\mathrm{s}}(\mathrm{dB})$ \\
\hline 63 & -2 \\
125 & -4 \\
250 & -10 \\
500 & -18 \\
1000 & -25 \\
2000 & -24 \\
4000 & -17 \\
8000 & -11 \\
\hline
\end{tabular}

